\title{
A FACTORIZATION THEOREM FOR LOGHARMONIC MAPPINGS
}

\section{ZAYID ABDULHADI and YUSUF ABUMUHANNA}

Received 24 March 2002

We give the necessary and sufficient condition on sense-preserving logharmonic mapping in order to be factorized as the composition of analytic function followed by a univalent logharmonic mapping.

2000 Mathematics Subject Classification: 30C55, 30C62, 49Q05.

Let $D$ be a domain of $\mathbb{C}$ and denote by $H(D)$ the linear space of all analytic functions defined on $D$. A logharmonic mapping is a solution of the nonlinear elliptic partial differential equation

$$
\overline{f_{\bar{z}}}=\left(a \frac{\bar{f}}{f}\right) f_{z},
$$

where $a \in H(D)$ and $|a(z)|<1$ for all $z \in D$. If $f$ does not vanish on $D$, then $f$ is of the form

$$
f=H \cdot \bar{G},
$$

where $H$ and $G$ are locally analytic (possibly multivalued) functions on $D$. On the other hand, if $f$ vanishes at $z_{0}$, but is not identically zero, then $f$ admits the local represenation

$$
f(z)=\left(z-z_{0}\right)^{m}\left|z-z_{0}\right|^{2 \beta m} h(z) \overline{g(z)},
$$

where

(a) $m$ is a nonnegative integer,

(b) $\beta=\overline{a(0)}(1+a(0)) /\left(1-|a(0)|^{2}\right)$ and therefore $\mathfrak{R} \beta>-1 / 2$,

(c) $h$ and $g$ are analytic in a neighbourhood of $z_{0}$.

In particular, if $D$ is a simply connected domain, then $f$ admits a global representation of the form (3) (see, e.g., [2]). Univalent logharmonic mappings defined on the unit disk $U$ have been studied extensively (for details, see, e.g., $[1,2,3,4,5,6])$.

In the theory of quasiconformal mappings, it is proved that for any measurable function $\mu$ with $|\mu|<1$, the solution of Beltrami equation $f_{\bar{z}}=\mu f_{z}$ can be factorized in the form $f=\psi \circ F$, where $F$ is a univalent quasiconformal mapping and $\psi$ is an analytic function (see [8]). Moreover, for sense-preserving 
harmonic mappings, the answer was negative. In [7], Duren and Hengartner gave a necessary and sufficient condition on sense-preserving harmonic mapping $f$ for the existence of such factorization. Since logharmonic mappings are preserved under precomposition with analytic functions, it is a natural question to ask whether every sense-preserving logharmonic mapping can be factorized in the form $f=F \circ \phi$ for some univalent logharmonic mapping $F$ and some analytic function $\phi$.

It is instructive to begin with two simple examples.

EXAMPLE 1. Let $f$ be the logharmonic mapping $f(z)=z^{2} /|1-z|^{4}$ defined on the unit disc $U$. Then $f$ is sense-preserving in $U$ with dilatation $a(z)=z$. We claim that $f$ has no decomposition of the desired form in any neighborhood of the origin. Suppose on the contrary that $f=F \circ \phi$, where $\phi$ is analytic near the origin and $F$ is univalent logharmonic mapping on the range of $\phi$. Then $F$ is sense-preserving because $f$ is. Without loss of generality, we suppose that $\phi(0)=0$. Then $F$ has a representation $F=\zeta H(\zeta) \overline{G(\zeta)}$, where $H$ and $G$ are analytic and have power series expansion

$$
H(\zeta)=\sum_{n=0}^{\infty} A_{n} \zeta^{n}, \quad G(\zeta)=\sum_{n=0}^{\infty} B_{n} \zeta^{n},
$$

where $\left|A_{0}\right|=\left|B_{0}\right|=1$.

Since the analytic part of $f(z)$ is $\phi(z) H(\phi(z))=z^{2} /(1-z)^{2}$, the function $\phi$ must have an expansion of the form

$$
\phi(z)=c_{2} z^{2}+c_{3} z^{3}+\cdots .
$$

It follows that $G \circ \phi$ has an expansion of the form $B_{0}+C_{1} z^{2}+C_{2} z^{3}+\cdots$. However, the given form of $f$ shows that $G(\phi(z))=1 /(1-z)^{2}=1+2 z z+$ $3 z^{2}+\cdots$, this leads to contradiction. Hence, $f$ has no factorization of the form $f=F \circ \phi$ of the required form in any neighborhood of the origin.

EXAMPLE 2. Let $f(z)=z^{2}\left|z^{2}\right|$ be defined in the unit disc $U$. Now, $f$ is sensepreserving logharmonic mapping in $U$ since the dilatation $a(z)=1 / 3$. But here $f$ has the desired factorization $f=F \circ \phi$, with $F(\zeta)=\zeta|\zeta|$ and $\phi(z)=z^{2}$.

Now, we state and prove the factorization theorem.

THEOREM 3. Let $f$ be a nonconstant logharmonic mapping defined on a domain $D \subset \mathbb{C}$ and let $a$ be its dilatation function. Then, $f$ can be factorized in the form $f=F \circ \phi$, for some analytic function $\phi$ and some univalent logharmonic mapping $F$ if and only if

(a) $|a(z)| \neq 1$ on $D$;

(b) $f\left(z_{1}\right)=f\left(z_{2}\right)$ implies $a\left(z_{1}\right)=a\left(z_{2}\right)$.

Under these conditions, the representation is unique up to a conformal mapping; any other represenation $f=F_{1} \circ \phi_{1}$ has the form $F_{1}=F \circ \psi^{-1}$ and $\phi_{1}=$ $\psi \circ \phi$ for some conformal mapping defined on $\phi(D)$. 
Proof. Suppose that $f=F \circ \phi$, where $F$ is a univalent logharmonic mapping and $\phi$ is an analytic function. Let $A(\zeta)$ be the dilatation function of $F$. Then simple calculations give that $f_{z}=F_{w}(\phi) \phi^{\prime}, f_{\bar{z}}=F_{\bar{w}}(\phi) \overline{\phi^{\prime}}$, and $a(z)=$ $A(\phi(z))$. Since $F$ is univalent, the Jacobian is nonzero and hence $|a(z)|=$ $|A(\phi(z))| \neq 1$ (see [2]). Also, $F$ is univalent and $f\left(z_{1}\right)=f\left(z_{2}\right)$ implies that $\phi\left(z_{1}\right)=\phi\left(z_{2}\right)$. Hence, $a\left(z_{1}\right)=a\left(z_{2}\right)$.

Next, suppose that the two conditions are satisfied. We want to show that $f$ can be factorized in the form $f=F(\phi)$. This is equivalent to finding a univalent continuous function $G$ defined on $f(D)$ so that $G \circ f$ is analytic. In view of the Cauchy-Riemann conditions, this is equivalent to

$$
\left(G_{w} b+G_{\bar{w}}\right) \overline{f_{z}}=0,
$$

where $b(z)=\overline{a(z)}(f(z) / \overline{f(z)})=f_{\bar{z}} / \overline{f_{z}}$.

Hence, $-b\left(f^{-1}(w)\right)=G_{\bar{w}} / G_{w}$. Let $\mu(w)=G_{\bar{w}} / G_{w}$. Now, we show that $\mu$ is well defined. Suppose that $f\left(z_{1}\right)=f\left(z_{2}\right)=w$. Then, as $b\left(z_{1}\right)=\overline{a\left(z_{1}\right)}\left(f\left(z_{1}\right) /\right.$ $\left.\overline{f\left(z_{1}\right)}\right), b\left(z_{2}\right)=\overline{a\left(z_{2}\right)}\left(f\left(z_{2}\right) / \overline{f\left(z_{2}\right)}\right)$, and $a\left(z_{1}\right)=a\left(z_{2}\right)$, it follows that $b\left(z_{1}\right)=$ $b\left(z_{2}\right)$. Hence, $\mu(w)$ is well defined and $|\mu(w)| \neq 1$ for all $w \in f(D)$.

Let $\left\{D_{n}\right\}$ be an exhaustion of $D, \Omega_{n}=f\left(D_{n}\right)$ and let $\mu_{n}$ be the restriction of $\mu$ to $\Omega_{n}$. Extend $\mu_{n}$ to $\overline{\mathbb{C}}$ by assuming that $\mu_{n} \equiv 0$ on $\mathbb{C} \backslash \Omega_{n}$. Then the Beltrami equation $G_{\bar{w}}=\mu_{n} G_{w}$ has a quasiconformal solution $G_{n}$ from $\mathbb{C}$ on $\mathbb{C}$, see [8]. Let $G_{n}(\infty)=\infty$, then $G_{n}$ is a homeomorphism on $\overline{\mathbb{C}}$. Replace the solution $G_{n}$ with the solution

$$
H_{n}(w)=\frac{G_{n}(w)-G_{n}\left(w_{0}\right)}{G_{n}\left(w_{1}\right)-G_{n}\left(w_{0}\right)},
$$

where $w_{0}, w_{1} \in \Omega_{1}$ and $w_{1} \neq w_{0}$. This is possible because $f$ is not constant on $D_{1}$. Then, $H_{n}$ is also a homeomorphic solution to the Beltrami equation, normalized to satisfy $H_{n}\left(w_{0}\right)=0, H_{n}\left(w_{1}\right)=1$, and $H_{n}(\infty)=\infty$. This and the fact that each $H_{n}$ is $K$-quasiconformal mapping on $\Omega_{j}$ imply that $H_{n}$ converges locally uniformly to a $K$-quasiconformal mapping $H$ on $\Omega_{j}$. It follows that $H$ is a homeomorphism on $\Omega$ and $H$ satisfies the equation

$$
H_{\bar{w}}=\mu H_{w} \quad \text { on } \Omega .
$$

Hence, $\phi=H \circ f$ is analytic in $D$.

Next, we show that $F=H^{-1}$ is logharmonic mapping. Note that $f=F \circ \phi$ was assumed to be logharmonic in $D$. Then, near any point $\zeta=\phi(z)$ where $\phi^{\prime}(z) \neq 0$, we can then deduce that $F=f \circ \phi^{-1}$ is logharmonic, where $\phi^{-1}$ is a local inverse. But $F$ is locally bounded, so the (isolated) images of critical points of $\phi$ are removable, and $F$ is logharmonic mapping on $\phi(D)$.

Finally, we prove the uniqueness. Suppose that $f=F \circ \phi=F_{0} \circ \phi_{0}$. If we let $G_{0}=F_{0}^{-1}$, then $G_{0} \circ f=\phi_{0}$ is nonconstant and analytic, and $G_{0 \bar{w}}=\mu G_{0 w}$. But the solution of this Beltrami equation is unique; hence, $G_{0}=G$. This completes the proof of the theorem. 


\section{REFERENCES}

[1] Z. Abdulhadi, Close-to-starlike logharmonic mappings, Int. J. Math. Math. Sci. 19 (1996), no. 3, 563-574.

[2] Z. Abdulhadi and D. Bshouty, Univalent functions in $H \cdot \bar{H}(D)$, Trans. Amer. Math. Soc. 305 (1988), no. 2, 841-849.

[3] Z. Abdulhadi and W. Hengartner, Spirallike logharmonic mappings, Complex Variables Theory Appl. 9 (1987), no. 2-3, 121-130.

[4] Univalent harmonic mappings on the left half-plane with periodic dilatations, Univalent Functions, Fractional Calculus, and Their Applications (Kōriyama, 1988), Ellis Horwood Ser. Math. Appl., Horwood, Chichester, 1989, pp. 13-28.

[5] _ Univalent logharmonic extensions onto the unit disk or onto an annulus, Current Topics in Analytic Function Theory, World Scientific Publishing, New Jersey, 1992, pp. 1-12.

[6] _ One pointed univalent logharmonic mappings, J. Math. Anal. Appl. 203 (1996), no. 2, 333-351.

[7] P. Duren and W. Hengartner, A decomposition theorem for planar harmonic mappings, Proc. Amer. Math. Soc. 124 (1996), no. 4, 1191-1195.

[8] O. Lehto and K. I. Virtanen, Quasiconformal Mappings in the Plane, 2nd ed., Springer-Verlag, New York, 1973.

Zayid Abdulhadi: Department of Computer Science, Mathematics and Statistics, American University of Sharjah (AUS), P.O. Box 26666, Sharjah, United Arab Emirates

E-mail address: zahadi@aus.ac.ae

Yusuf Abumuhanna: Department of Computer Science, Mathematics and Statistics, American University of Sharjah (AUS), P.O. Box 26666, Sharjah, United Arab Emirates

E-mail address: ymuhanna@aus . ac . ae 


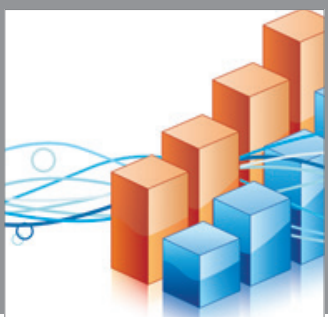

Advances in

Operations Research

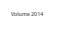

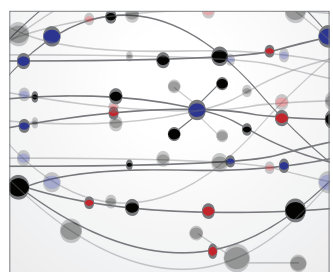

\section{The Scientific} World Journal
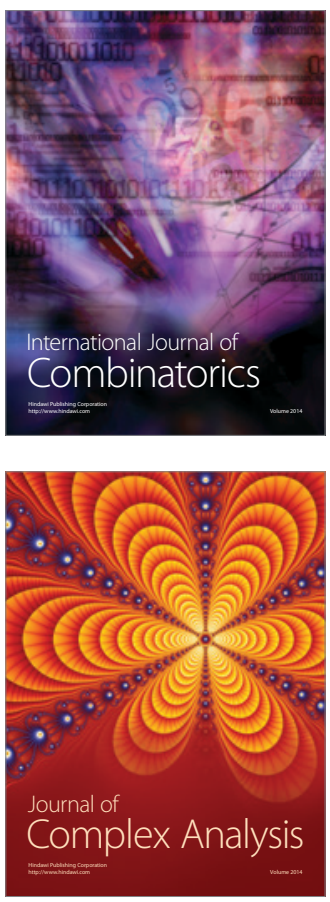

International Journal of

Mathematics and

Mathematical

Sciences
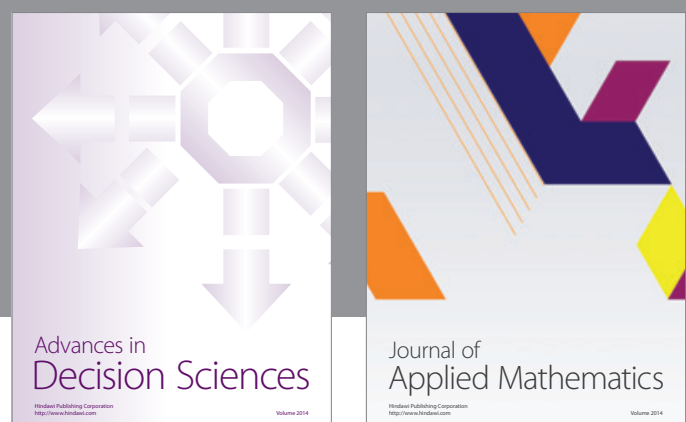

Journal of

Applied Mathematics
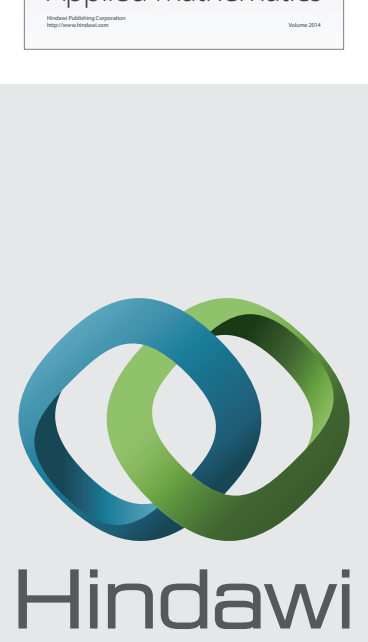

Submit your manuscripts at http://www.hindawi.com
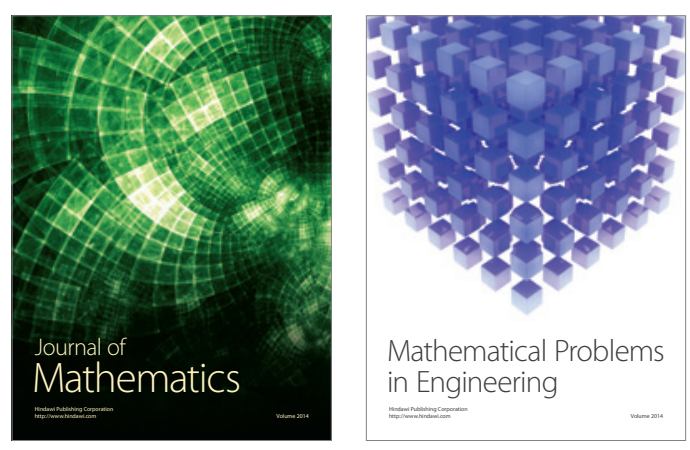

Mathematical Problems in Engineering
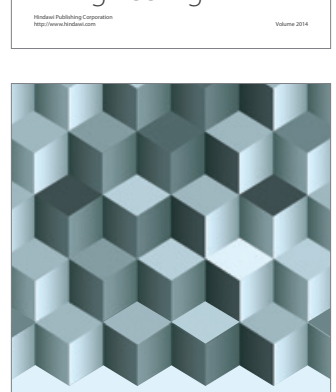

Journal of

Function Spaces
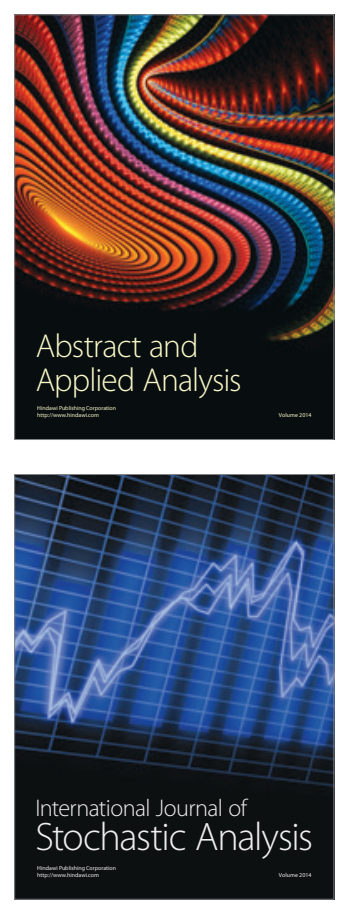

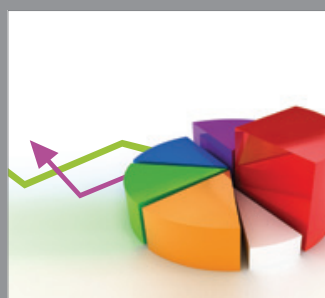

ournal of

Probability and Statistics

Promensencen
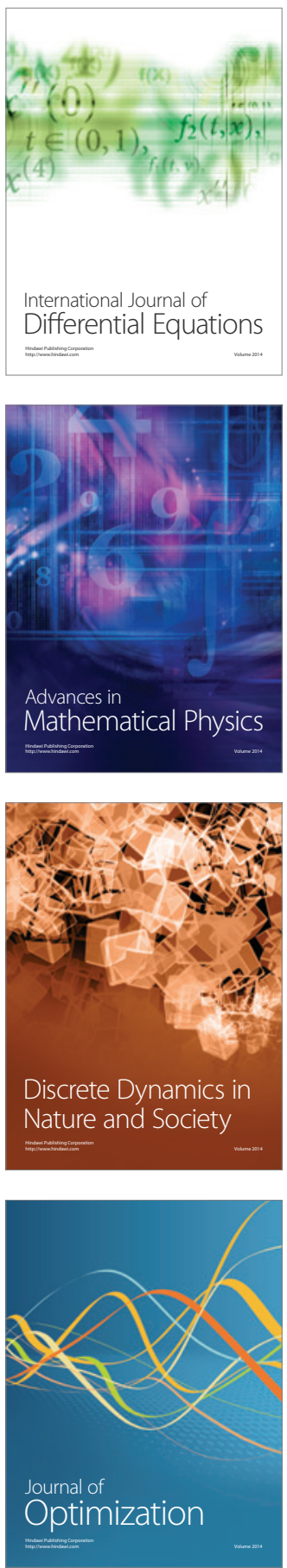